\title{
Disaster Management in India: A Road Ahead
}

\author{
Sonal Pruthi; ${ }^{1}$ Amitesh Aggarwal, MD $;^{2}$ Ashish Goel, $\mathrm{MD}^{2}$
}

1. University College of Medical Sciences, Delhi, India

2. Department of Medicine, University College of Medical Sciences, Delhi, India

\section{Correspondence:}

Sonal Pruthi

University College of Medical Sciences

Dilshad Garden

Delhi, India

E-mail: sonalpruthi@hotmail.com

doi:10.1017/S1049023X12001616
Heartbeats taken too soon amongst silent prayers, debris scattered for miles, houses unrecognizable, treasures lost amidst the trampled smell of mud, where broken chairs and teddy bears lie... an apocalyptic nightmare meets the eye after a disaster.

The great Indian Ocean tsunami of 2004, with a death toll of 300,000, wiped out civilization in many parts of Southeast Asia. Where at the start of the day, people were going about their normal lives, at the end of the day millions were struggling with reality of tens of thousands of dead or missing relatives, destroyed homes, and shattered lives. The thousands of corpses hanging on trees or washed up on beaches immediately started to rot in the tropical heat, and exposed the pitiful, ragged state of disaster management system in India.

At a time of global changes, it is vital that India pays attention to its disaster management facilities. Disaster management is a continuous process by which individuals, groups and communities manage hazards in an effort to ameliorate the impact of disaster. Due to the country's unique geoclimatic location, $60 \%$ of the landmass is prone to earthquakes; $68 \%$ to drought; eight percent to cyclones and $12 \%$ to floods.

India has experienced many major natural disasters in the past few years, which has made us realize that the need of the hour is to adopt a multi-dimensional, multi-disciplinary and multi-sectoral approach to create a robust system which can create awareness, efficiently prevent disaster and manage once the disaster has taken place. Though government has taken baby steps in that direction, there is a yawning gap between what is planned and what is implemented.

The need of the hour is an integrated effort to collect and compile data, including information and local knowledge on disaster history and response patterns. Another need is an Early Warning System to provide timely information, targeting the important minutes before disasters when measures can be taken to minimize damage.

Another requirement is a reliable and cost-efficient telecommunications link for humanitarian relief and assistance agencies, along with a portable telemedicine system with satellite connectivity. Equally important is to keep in mind the vulnerability of critical infrastructure. Hence, agencies should be equipped with electrical and solar generators, lighting equipment, fuel, solar cookers and other technologies to take care of power supply and basic needs. Safe water availability with low-cost, local treatment facilities is important during post-disaster situations for sanitation and to avoid outbreak of other epidemics.

Manpower is of vital importance. It is imperative to train personnel for effective triage and first aid - the lack of training is a prime concern. Equally relevant is to have regular practice drills to train the population to assist in relief activities in the event of a disaster. Sustainability of efforts is critical. Unless the systems are maintained in good condition, all efforts are futile.

To summarize, we must take an integrated approach to create a Culture of Disaster Preparedness and Prevention, in which people at risk receive, understand, and act upon the warning information conveyed. We realize that though the unexpected can happen at any time and we cannot control the unexpected, we can control how we plan and respond. If sufficient number of management layers are superimposed, it can be assured that disaster is not left to chance. 